\title{
Discussion on the Painting Language of Acrylic Material in Modern Painting
}

\author{
Lijun Yi \\ Sichuan Fine Arts Institute \\ Chongqing, China
}

\begin{abstract}
Acrylic material is the crystallization of the development of modern science and technique, and its emergence has promoted the development of modern painting art to a certain extent, enriched the expression language of painting. This paper discusses the painting language of acrylic material in modern painting, introduces the development of acrylic material painting, the painting language characteristic of acrylic material, and the expression technique of acrylic painting material.
\end{abstract}

Keywords-modern painting; acrylic material; painting language

\section{INTRODUCTION}

Painting language refers to the mode of expression in which the painters create artistic image to form visual experience through modeling means for the purpose of recording the current state, reflecting and conveying information or expressing ideas, with material tools such as pigment, paintbrush, knife and paper as characteristic carrier, and visual signals such as color, lines, graphics and texture as media. (2) It is the created visual symbol, visual signal and visual language.

Acrylic pigment is a new kind of paint pigment mixed by a chemical synthetic rubber emulsion (containing acrylic ester, metacrylic acid ester, acrylic acid, methyl methacrylic acid, thickening agent and filer) with color particles.

Acrylic painting refers to the painting taking synthetic acrylic resin added with thickening agent and filler as the media, mixed with color particles into pigment. The components of the acrylic colors used are "dispersible pigments made from polymethyl methacrylate dissolved in mineral spirits, sometimes referred to as pure acrylic or plastic pigments, to be distinguished from polymeric material containing propylene and other water-soluble resins. In Chinese Dictionary of Arts and Crafts there is the following description: "Its main component is polyacrylate latex. It has the characteristics of bright color, strong adhesion, fast drying, strong water resistance and so on. It can be used to draw a variety of types of paintings on multiple carriers (such as plastic film, cloth, paper, wood, bamboo, shell, lead skin, cement, etc.)."

\section{RESEARCH ON THE DEVELOPMENT AND APPLICATION OVERVIEW OF ACRYLIC MATERIAL PAINTING}

The study of the combination of acrylic materials and pigment originated in Germany at the beginning of the 20th century. "In 1880, Germany first reported the hard transparent solid substance that can be polymerized by methyl acrylate". With the development of petrochemical industry, especially in the First World War, the German scientist Lemu developed acrylic the industrial manufacturing method of acrylic ester, "in 1928 he established ROHM - Haas Company which took acrylic monomer and polymer as main products in the United States. In 1932, Imperial Chemical Industries established the industrial manufacturing method of methacrylic acid. In recent years, the direct oxidation to acid acrylic from propylene has been achieved, so that the scale of production is expanded. The acrylic ester's price is cheaper, with a wider application area" . (2) The progress of science and technique and the development of new painting materials promote the development of painting materials disciplines.

In 1935, through experiment, Siqueiros found that the wet fresco techniques from Europe was not able to adapt to the modern construction built with reinforced concrete, and a new painting material with strong adhesion, light fastness and stable nature must be found. Siqueiros established Siqueiros Painting Materials Experiment Studio in New York, USA. Painters and scientists cooperated closely, and after several tests with emerging polyvinyl acetate emulsion as medium, which contributed to the generation and development of acrylic pigment. The Mexican fresco movement in the 1930s was a painting movement on the wall of the capital of Mexico and other cities on the basis of people's life and struggle. It was a cultural revolutionary movement under the influence of bourgeois democratic revolution. "(3) Under its influence and advocacy, the acrylic material was first applied to the painting field, and acrylic material painting suddenly rose and sparkled in the art field with its unique performance.

After World War II, art activities with New York as the art center were unprecedentedly prosperous. In the 1950s, American abstract painter Pollock took the lead in using acrylic paints for experimental creation. Many artists also used new materials to create, keeping pursuing original and unique forms of artistic expression, and new art schools appeared successively. "Abstract Expressionism" became one of the most representative genres of artistic style. Representative 
painters, including Pollock, Motherwell, De Kooning, Hoffman, Mark Toby, created a large number of works with propylene, exerting a great influence on the development of modern American painting, strongly promoting the spread of acrylic material painting in the world.

China began to develop acrylic resin in 1960s, in 1982, Shanghai Maries Painting Material Company began to produce acrylic pigment, so far, the production technique and product quality are improving. "In 1997, the Central Academy of Fine Arts and the United States of America San Francisco Academy of Fine Arts ran a school jointly, opening workshop on western painting new materials, in which Jamie Mogeng from America San Francisco Academy of Fine Arts and Richard Rodrigo from the Art Department of San Francisco City University served as keynote speakers. (4) They introduced the latest acrylic pigment products in the United States, exerting a great influence on the domestic art circle, and many art colleges successively opened relevant courses. Many artists painted with acrylic materials which had become an important member of the field of painting and related fields.

\section{ANALYSIS OF THE ACRYLIC MATERIAL'S PAINTING LANGUAGE CHARACTERISTICS}

\section{A. The Water-Based Characteristics of Acrylic Material Painting}

1) Flow and fusion: The material painting has several aspects of performance, which requires the pigment matrix particles be be fine and easy to dissolve in water; to flow, fuse, precipitate and be transparent. Acrylic materials have excellent performance. It's basic painting technique refers to watercolor painting language, especially the application of thin coating technique in painting process combines with the appropriate proportion of water, which can exert the characteristics of flow, transparency, fusion and volatility. It can both depict finely and also render and mask with freehand brushwork and transparency. According to the characteristics of water-based materials, it combines with the needs of the picture to appropriately exaggeratively moisten, fuse and permeate, and the completed picture blends water and color, with vivid charm. Especially the effect of occasional flow and fusion of water has been effectively controlled and exerted to improve the artistic expression of the work. The characteristics of water-based materials widen the performance field of acrylic painting, and enrich and diversify the artistic styles.

2) Rendering and masking: Traditional Chinese painting techniques in China has a long history, with various schools and numerous masters, and deep research. The "hooking, cracking, stippling, rendering" in Chinese painting techniques stress pen and ink use. The color pays attention to coloring according to type. Xie He in the Southern Dynasties stressed in the "A Record of Comments on Ancient Paintings": "vivid charm" and "coloring according to type". Coloring of flowers in fine brushwork paid attention to the inherent color and inherent color brightness changes of the image. The coloring category is divided into: ink color, light color, pastels and strong coloring. The specific coloring method is flat coating, rendering, uniform rendering, masking, 3D toner, water accumulation, skeletonless method, setting off by contrast and so on. There is also mixed cross-use of techniques such as flowing, leaving blank, spraying in watercolor painting techniques, and acrylic painting materials enhance the dripping rendering of water-based materials through the absorption the integration of Chinese painting techniques and watercolor painting techniques; implement plastic and oily masking techniques, enrich the painting language of acrylic material, and enrich and diversify the performance techniques of painting.

3) Separation and overlaying of water characteristics:An important feature of the material is the proper control of moisture. Acrylic pigment particles are very fine, and can become crystal clear when dissolving in water, especially with the use of Tampera indirect techniques, pigments form a small "lake" on the picture, swimming and fusing, color blended, thus changing the original color state, producing the effect of color overlaying, so that the color becomes warm and deep. When the picture is $60 \%-70 \%$ dry, spray alcohol and diluted glue on the color layer to separate and permeate between the pigment and base and form a variety of color differentiation effects. Separation and overlaying make the picture create a new complementary relationship and color-shaped mark, and become and important component of the picture.

\section{B. Language Compatibility of the Material}

In the 1970s, the exploration of the language of painting material in contemporary art turned to the exploration of the characteristic language of the material itself, to the perception and understanding of the painting language and explored its language extension. Modern painting turned comprehensive, and many artists had their own unique techniques, with a variety of uses of painting materials. The generation of acrylic materials is compatible with more linguistic approaches. The creation of a work could start from the classical Tampera or classical oil painting as base, go through white sketch, waterbased material rendering, acrylic material modeling, and finally end in the oil painting, in which both Chinese painting image rendering and watercolor transparent thin coating could be used, and heavy texture treatment like oil painting could be applied. As long as it followed the basic principle of "plumpness covering thinness", it could achieve the effect of combining advantages of techniques of different material painting. Acrylic material painting and water-based Tampera, watercolor, gouache, ink and wash painting all belong to water-based material painting system, with a certain degree of compatibility, which is compatible, flexible and has unique charm, transcending many limitations of traditional paintings. The use of acrylic material makes the painting process full of randomness and contingency, whose unique materiality of the material makes the picture language full of symbols and meanings, and enriches the painting language in the balance of abstract language and material beauty. 


\section{The Comprehensive Nature of the Material}

1) Compatibility of techniques: Compared with traditional pigments, acrylic material has great inclusiveness as a product of modern technique, showing a stronger language performance force in application of collage, mixed technique and water-based Tampera technique in the field of composite material art form. It makes the material painting truly compatible and integrated, which is soluble in both water and oil, suitable for painting on a variety of material surfaces. In performance techniques, acrylic pigment also shows great advantages: transparency of watercolor, heaviness of oil painting, covering of gouache, color overlapping of Tampera, image rendering of traditional Chinese painting and polishing of lacquer painting can almost all be achieved by acrylic material, which is the pigment with greatest modern significance in current painting material system. For example, the American painter Jean-MichelBasquiat's representative work "Unti-tled" was done in 1984 with propylene, silk and oil paint, the cloth size is $223.5 \times 195.6 \mathrm{~cm}$. "Hastily sketched characters, handwritten text and scientific digital schema, together with rich color, form a visual experience whose color and form is disharmonious. The original, innocent image reflects Basquiat's relevance to graffiti art. The whole picture, with its unconnected image and handwritten inscription, seems to be the spirit of New York lower society in which the painter rooted, which evokes the multi-ethnic and hippie culture, and also reflects the real situation of rapid changes of city life and chaos". The material used in this painting, propylene, silk picture, oil painting, a mixture of a variety of techniques, was a feature of the art boom in 1980s.

2) Compatibility of form: The comprehensive material began to go into the field of painting from the collage, representative cubism painters such as Picasso, Braque and Matisse implemented bold exploration and experimentation in the structural form of comprehensive materials, enriched the formal language of painting. Polyacrylic acid ester has features of strong adhesion, fast drying, strong water resistance and so on. The representative work, Picasso's "Cards Player" $108 \times 89.5 \mathrm{~cm}$, was collaged with wood, newspapers, plastic and other materials. It achieved contradictory exchange and impact between many raw materials with different materials, so as to bear the combination, collage and reconstruction of pigment and real object, with a new look. When the art history developed to the Cubism period, the artists' collage, reconstruction, mix-andmatch of the form language of comprehensive material had become a natural choice in the their creation. It became an important symbol of a certain style of artists, and had an independent aesthetic value. From the beginning, futurism, surrealism and Dadaism, puristism, supremacy, constructivism had widely experimented on the use of collage and comprehensive materials.

3) Compatibility of meaning: The concept and meaning conveyed by excellent art works always have a similar feature, that is to keep influencing and changing people's aesthetic methods and visual experience. Since the art of composite material painting went on the stage of history, the aesthetics of material significance can also be used as one of the important criteria of evaluation of the picture quality, which shows us a mysterious new world of art vision with rich connotation, and many artists have created classic works in the application of new materials and exploring expression of the material spirit, such as Rauschenberg, Brie, David Salisbury, Kiefer, David Hockney. The openness of acrylic material painting and its compatibility of meaning show great advantages in collage and comprehensive material art. With the recombination of existing collage, with dream, illusion, reality, symbol and metaphor, the original image content turns to new vocabulary in the new work context, and the recipient unconsciously participates in the re-perception in creation, so that the concept and meaning of works are extended.

\section{EXTENSION OF PAINTING LANGUAGE OF ACRYLIC MATERIAL}

Materials are applied widely. More and more materials begin to be applied to the picture comprehensively, making the components of the painting language more numerous and complex. The material itself is a unique painting language. Acrylic material as a new type of painting material, is the product of high-tech era, and the production of acrylic painting materials promotes the development of contemporary art. Acrylic material painting broadens the visual field of art, which is a perfect crystallization of science and art. Acrylic materials can be used in combination with other materials and techniques to create a variety of expression languages, which can present a variety of effects on a sheet of paper. Artists can furthest show their creativity and emotion without being limited by material techniques. The art work created through integration of multiple materials often show the artistic effect which can not achieved by other materials, and such works thus also form a unique style. More and more contemporary artists have take it as the ideal material to express their ideas, which continues expanding and extending the use of acrylic materials in painting and related fields. In the context of contemporary art, many artists study and use new materials, and the material media begin to move from the behind-thescenes of artistic language to the foreground and become the ontological language of art works. Unique language features expand the artistic expression space for us. The painting with acrylic material as medium has been used in combination with many more complicated media, which has a positive significance to the exploration of the acrylic material painting in the contemporary context.

\section{CONCLUSION}

At present, the artists are still a new exploration on the characteristics and application experiment of acrylic painting, and the continuous progress of material science promotes the improvement of new materials. After improvement, the unique beauty of the acrylic material painting will become increasingly prominent. There will be more and more artists using acrylic materials for creation, and the rise of acrylic material painting classic works has been just around the corner. 


\section{REFERENCES}

[1] Sun Hongchen. The Origin and Development of Comprehensive Material Painting [J]. Art Ocean, 2011 (8): 73

[2] Ma Xinping, Zhang Hailan. Combination and Application of Acrylic Pigment and Tampera Technique [J]. Art Education, 2015 (1): 201-202

[3] Ma Xiaoqing. Liu Youju. Primary study on painting language impression on Liu Youju Illusionist Painting Exhibition in Beijing [J]. Collectors, 2015 (5): 144-145 